\title{
THE EFFECT OF CYCLIC NUCLEOTIDES ON VIRUS INFECTION*
}

\author{
H. F. Maassab and R. M. Soltysiak† \\ Department of Epidemiology \\ School of Public Health \\ The University of Michigan \\ Ann Arbor, Michigan 48109
}

Cyclic nucleotide research, from the standpoints of both organic chemistry and mode of action in the physiology of the mammalian cell, remains a relatively new field. Cyclic AMP was first described by Sutherland and Rall' ${ }^{\prime}$ as the mediator of the hepatic glycogenolytic effect of epinephrine and glucagon and is now recognized as a versatile regulatory agent mediating a host of hormonal effects. Research has uncovered a vast pool of potential effects of the cyclic nucleotides in cellular differentiation, dedifferentiation, and transformation, all of which implicate various enzymes, protein synthetic mechanisms, and viruses.

Accumulation of intracellular cAMP can be initiated and accelerated by plasma membrane adenyl cyclase, at the expense of ATP pools. A classic example is that of hormonal activation of the "receptor" subunit of adenyl cyclase in adrenergic fashion. Whereas the influence of $\beta$ receptors may be positive in increasing the activity of the catalytic adenyl cyclase subunit, $\alpha$ receptors may mediate a reduction in activity. ${ }^{2}$ By such means, cAMP levels are modulated by the net effects of a stimulus. On the other hand, specific cellular phosphodiesterases, two types of which are known to exist in cultured mammalian cells, each differing in $K_{\mathrm{M}}$ for cAMP, ${ }^{3}$ hydrolyze cAMP to the inactive adenosine 5'-monophosphate. Cyclic AMP exerts its effects by binding to a cAMP-specific receptor site on any of the various classes of protein kinases. ${ }^{4}$ The final effector step is presumably accomplished by utilization of enzymatic or structural proteins as substrate for phosphorylation by the activated kinase. The cellular regulatory effects ascribed to cAMP via DNA synthesis, transcription of mRNA, and the translation of such messengers may be understood in terms of phosphorylation of nuclear proteins, sigma-like factors, and ribosomal protein, respectively. Cyclic AMP addition is known to play a major role in many other metabolic functions in living cells. The level of cAMP in certain cells is believed to act in the regulation of the active transport of amino acids ${ }^{5,6}$ and thereby influence the rate of protein synthesis. Intracellular cAMP is also implicated in effecting the transport of water and electrolytes across a number of polar tissues. ${ }^{7}$ Given the widespread occurrence of cAMP-mediated regulatory phenomena both in animal cells and bacteria, we initiated the studies to explore (1) the role that cAMP plays in the development of a selected group of viruses, and (2) to ascertain whether observed changes in cAMP metabolism are related to expression of the viral genome.

*This work was supported in part by Grants 340475 and 320413 from the Cancer Research Pilot Project Research Fund.

† In partial fulfillment of the requirements for the degree of Doctor of Philosophy in Epidemiologic Science. 
Enhancement of Influenza Virus Growth by a Cyclic Nucleotide

\section{Effective Dose Level}

Type $A$ influenza A/AA/6/60 $\left(\mathrm{H}_{2} \mathrm{~N}_{2}\right)$ was plaque purified and pools were made by growing the virus in 11-day embryonated eggs at $33^{\circ} \mathrm{C}$. Procedures used to prepare primary chick kidney cells (PCKC) have been described elsewhere. ${ }^{8}$ Monolayers of these cells were established in 5 days using growth medium 199 with $10 \%$ calf serum.

The growth media of the established chick kidney monolayer was discarded, the cells were washed once in prewarmed Hank's balanced salt solution, and the virus inoculum was allowed to adsorb for $1 \mathrm{hr}$ at room temperature. Residual virus was removed, the cell sheet washed with Hank's balanced salt solution, and fresh maintenance media composed of double-strength Eagle's basal solution, but no serum, was added.

Work was initiated with $3^{\prime}: 5^{\prime}$-cyclic adenosine monophosphate (cAMP). However, its derivative $N^{6}, O^{2}$-dibutyryladenosine- $3^{\prime}: 5^{\prime}$-cyclic monophosphate (dcAMP) was substituted when published reports showed that its lipolytic properties made it more permeable for the cell membrane ${ }^{9}$ and that it inhibits phosphodiesterase catalyzed breakdown of cellular cAMP. ${ }^{10}$

The effect of dcAMP on influenza virus multiplication in primary chick kidney cells (PCKC) was followed by determining virus yield in untreated cells and in those treated with different doses of dcAMP. TABL.E I shows the results of a typical experiment. It is evident that enhancement in hemagglutinin (HA) yield is apparent at $24 \mathrm{hr}$ postinfection (p.i.), with either $25 \mathrm{mM}$ or $5 \mathrm{mM}$ concentrations when compared to yield from untreated cells. The results at $48 \mathrm{hr}$ p.i. were similar in both groups.

In general the compound was added just before inoculation of PCKC with $10^{4}$ $\mathrm{TCID}_{50} / \mathrm{ml}$ of Type $\mathrm{A}$ influenza virus $\left(\mathrm{A} / \mathrm{AA} / 6 / 60-\mathrm{H}_{2} \mathrm{~N}_{2}\right.$ ). Pretreatment of cells for up to $24 \mathrm{hr}$ prior to infection did not seem to alter appreciably the effect of dcAMP on the course of infection. Under similar conditions, enhancement of HA production was also apparent upon infection of PCKC with Type $B$ influenza virus (B/Palau/3/71).

\section{Effect of Virus Dose}

Since the enhancing effect of dcAMP was followed at only one level of virus inoculum, studies were undertaken to compare the effect of the dose of influenza virus on the yield of HA in treated and untreated PCKC. Results presented in TABLE 2 demonstrate clearly that the enhancing effect of dcAMP on the yield is dose-dependent. At $10^{-2}$ dilution of influenza virus pool (corresponding to a multiplicity of $10: 1$ ), there was no significant difference in the yield between control and dcAMPtreated PCKC. However, at $10^{-3}$ dilution, there is a definite increase in HA yield in dcAMP-treated cells as compared to untreated cells at $48 \mathrm{hr}$ p.i. At $10^{-4}$ and $10^{-5} \mathrm{di}-$ lution of influenza-virus, a significant enhancement in $\mathrm{HA}$ yield is apparent in dcAMP-treated cells at both 24 and $48 \mathrm{hr}$ p.i.

\section{Serial Passage with dcAMP}

Influenza virus was passed serially in treated and untreated PCKC holding constant both the concentration of compound $(2.5 \mathrm{mM})$ and the dilution of virus 
TABLE 1

EfFect of Various Concentrations of Dibutyryl camp (dcAMP) on Growth of INFLUENZA VIRUS (A/AA/6/60- $\mathrm{H}_{2} \mathrm{~N}_{2}$ )

\begin{tabular}{cccc}
\hline \hline & \multicolumn{3}{c}{ Hemagglutinin Titer $(1 / n)$} \\
\cline { 2 - 4 } Concentration of dcAMP, in mM & $0 \mathrm{hr}$ & $24 \mathrm{hr}$ & $48 \mathrm{hr}$ \\
\hline None (control) & $<2$ & 2 & 128 \\
100 & $<2$ & 64 & 256 \\
25 & $<2$ & 32 & 256 \\
5 & $<2$ & 4 & 256 \\
0.5 & $<2$ & 2 & 256 \\
0.05 & $<2$ & 2 & 128 \\
0.005 & & 2 & 2 \\
\hline
\end{tabular}

$\left(10^{-4}\right)$. The design of the experiment was to pass the virus every $48 \mathrm{hr}$ p.i. and use the yield to initiate the next passage. Results of the rapid serial passages of the A/AA/6/60- $\mathrm{H}_{2} \mathrm{~N}_{2}$ strain is illustrated in FIGURE 1. It is quite evident under the planned experimental design that upon serial passage in untreated cells, the virus yield was not enough to initiate the 3 rd passage and virus could not be detected by hemagglutinin. However, in the dcAMP-treated cultures due to enhancement reported earlier, the virus persisted and vigorous growth was evident throughout serial passage. Infectivity yield, as shown in Figure 2, was sustained in treated cells but declined sharply, and eventually no infectious virus was detected in the untreated cells. In addition the neuraminidase activity of influenza virus was also enhanced in the dcAMP-treated cells as compared to the untreated cells.

The amplification of growth of influenza virus has substantiation on the basis of replication of certain RNA tumor virus-like particles in cell culture. For instance, it was shown that treatment of Chinese hamster cells with dcAMP led to formation of typical C-type particles containing 70-S RNA and an RNA tumor virus-like, RNA-directed, DNA polymerase activity. "Treatment with the dcAMP did increase C-type particle formation in mouse leukemic $\mathrm{L} 5178 \mathrm{Y} / \mathrm{R}$ lymphoblast $4 \mathrm{hr}$ after start of treatment, this because of an apparent increase in the rate of viral maturation rather than induction. ${ }^{12}$

TABLE 2

Effect of Dose of Virus on Yield of Influenza in Chick Cells Treated with dcAMP*

\begin{tabular}{|c|c|c|c|c|}
\hline \multirow[b]{3}{*}{ Final Virus Dilution* } & \multicolumn{4}{|c|}{ Hemagglutinin Titer $(1 / n)$} \\
\hline & \multicolumn{2}{|c|}{ Cont rol } & \multicolumn{2}{|c|}{ dcAMP } \\
\hline & $24 \mathrm{hr}$ & $48 \mathrm{hr}$ & $24 \mathrm{hr}$ & $48 \mathrm{hr}$ \\
\hline $10^{-2}$ & 64 & 256 & 128 & 256 \\
\hline $10^{-3}$ & 16 & 64 & 64 & 256 \\
\hline $10^{-4}$ & 4 & 16 & 32 & 128 \\
\hline $10^{-5}$ & $<2$ & 2 & 8 & 64 \\
\hline
\end{tabular}

${ }^{*}$ The initial inoculum had a hemagglutinin titer of 256 and infectivity of $10^{\times .5} \mathrm{EID}_{50} / \mathrm{ml}$, where $E I D_{50}$ is the mean egg infectious dose. 


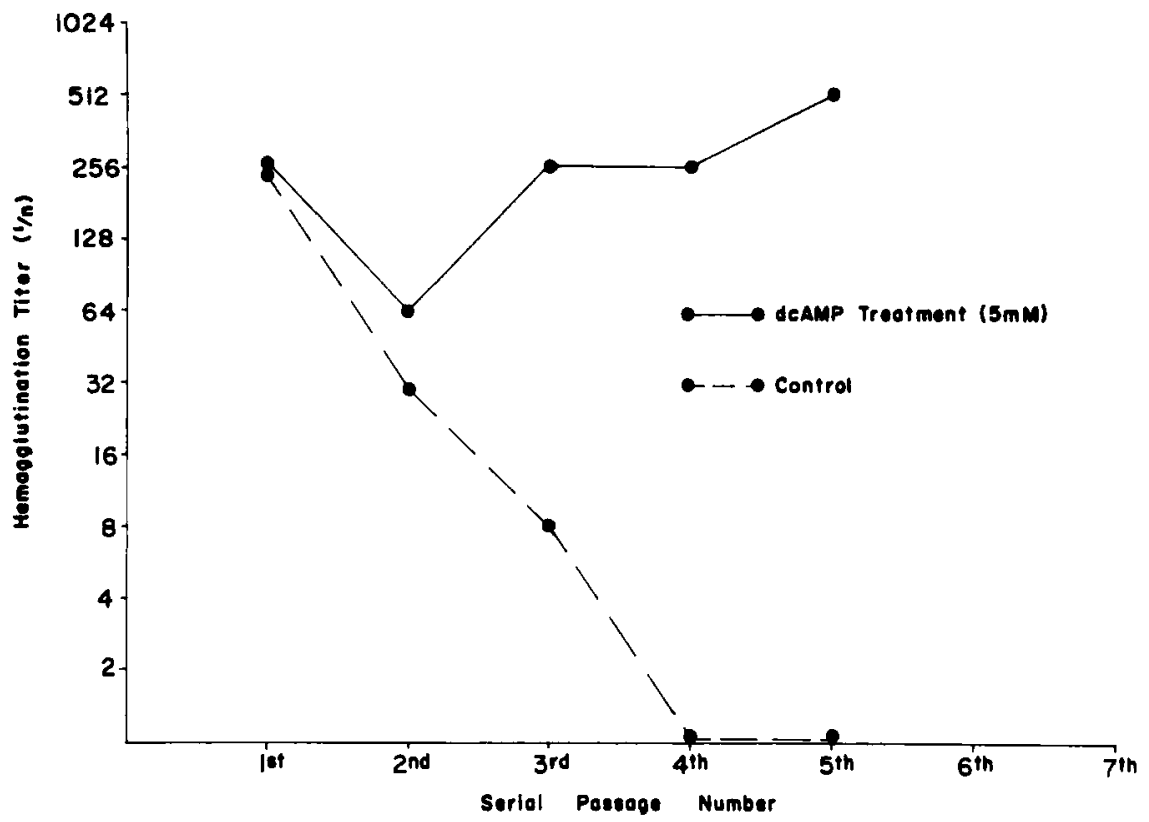

FIGURE 1. Serial passage of influenza virus in primary chick kidney cells (PCKC).

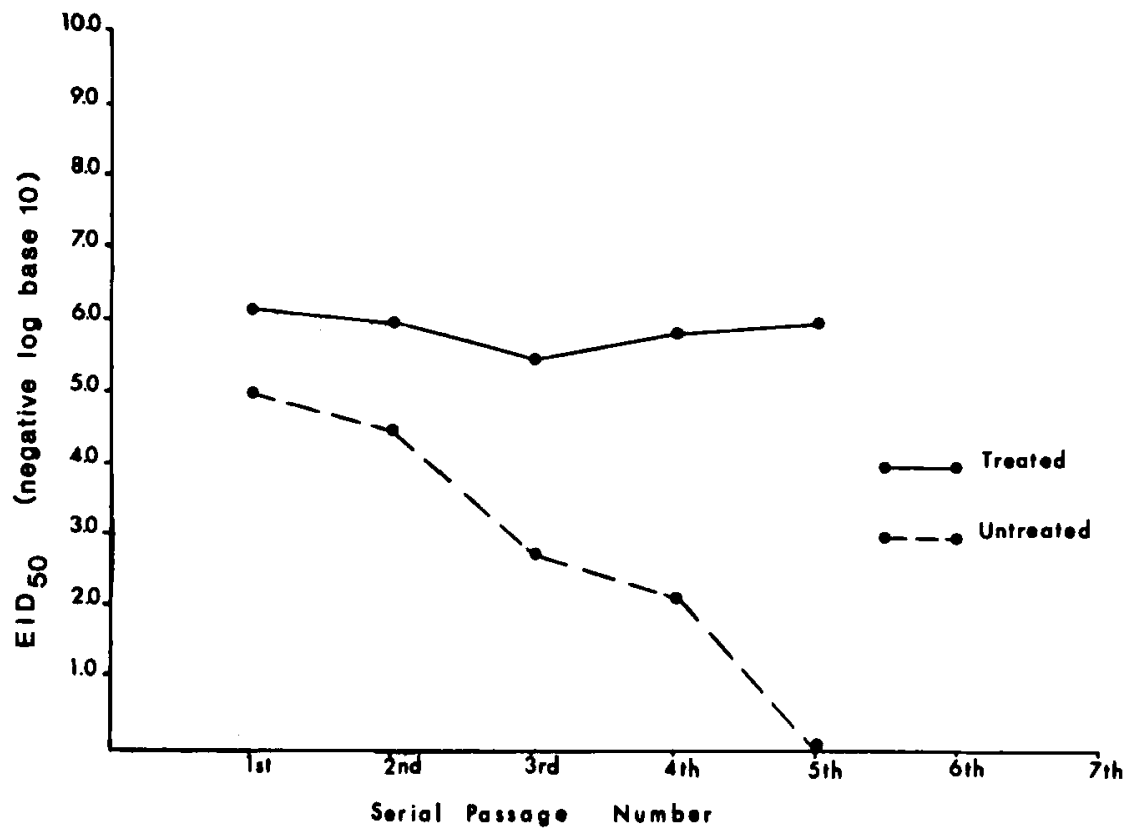

Figure 2. Infectivity yield of influenza virus after serial passage in chick cells. EID $D_{50}$ is the mean egg infectious dose. 
TABLE 3

Influence of dcamp on Isolation of Influenza Virus from Clinical Specimens*

\begin{tabular}{crcccccc}
\hline & \multicolumn{3}{c}{ PCKC-treated } & & \multicolumn{3}{c}{ PCKC-untreated } \\
\cline { 2 - 4 } \cline { 5 - 7 } Patient Name $\dagger$ & HA & HAD & CPE & & HA & HAD & CPE \\
\hline B.T. & 64 & $4+$ & $3+$ & & $1+$ & 0 \\
D.H. & 96 & $4+$ & $4+$ & & $1+$ & 0 \\
A.S. & 24 & $3+$ & $3+$ & 0 & $1+$ & 0 \\
C.H.. & 4 & $2+$ & $1+$ & 0 & 0 & 0 \\
\hline
\end{tabular}

*PCKC, primary chick kidney cells; HA, hemagglutination; HAD, hemadsorption; CPE, cytopathic effects.

† Specimens chosen on the basis that either no isolation resulted upon inoculation of embryonated eggs, or that two blind passages were required before positive isolation.

$\ddagger$ Influenza infection was documented by serological rise.

\section{Isolation Frequency of INFLUENZA Virus By dcAMP}

Amplification of influenza virus multiplication was also evident by the increase in frequency of isolation of influenza virus from nasopharyngeal swabs obtained from cases of clinical influenza as illustrated in TABLE 3 . Isolation frequency in permissive cell cultures, such as primary chick kidney or primary monkey kidney cells was increased by treatment with dcAMP. Not only can the rate of isolation be increased by $30 \%$, but also the time-course of incubation can be considerably shortened to approximately $24 \mathrm{hr}$ after inoculation of clinical specimens. Hemadsorption and hemagglutination are evident during this short interval in the dcAMP treated cultures only, which is of tremendous advantage in terms of rapid isolation and identification in terms of influenza outbreaks.

\section{Host-Dependent ENHancement}

Results presented in TABLE 4 show that the enhancement of growth of influenza virus was reproducible in all permissive primary cell lines tested, but not in nonper-

TABLE 4

EFFEct OF deAMP ON VIRAL GROWTH IN Different Hosts

\begin{tabular}{lcccc}
\hline & \multicolumn{2}{c}{ Influenza } & \multicolumn{2}{c}{ Sendai(HA)3 } \\
\multicolumn{1}{c}{ Host } & Treated & Nontreated & Treated & Nontreated \\
\hline PCKC* & 128 & 32 & 16 & 12 \\
Vero (AGMK) & NP\$ & NP\$ & 2 & 2 \\
LIC-MK ${ }_{2} \dagger$ & NP\$ & NP\$ & 256 & 256 \\
PAGMK $\ddagger$ & 32 & 2 & 16 & 32 \\
WI-38 & $48 \pi$ & 489 & NT $^{* *}$ & NT $^{* *}$ \\
\hline
\end{tabular}

*Primary chick kidney cells.

†Established rhesus monkey cell line.

$\ddagger$ Primary African green monkey kidney.

$\S \mathrm{NP}$, nonpermissive.

ILost upon subsequent passage.

${ }^{* *}$ NT, not tested. 
missive continuous cell lines. In general, primary cultures, which usually display density-dependent inhibition of growth, have an increase in cAMP levels at confluency, whereas continuous aneuploid cells have a decrease in cAMP levels at high density. Hence, it is possible to speculate that in heteroploid cell lines, the nonpermissive state can be related to the level of intracellular cAMP, which is definitely lower and does not fluctuate appreciably during cellular growth or by the exogenous addition of cyclic nucleotides.

In addition, the data illustrated in TABLE 4 show that addition of dcAMP to different permissive hosts infected with parainfluenza virus Type 1 (Sendai strain) did not result in any alteration of growth of the virus, since no enhancement or inhibition was demonstrated. Serial passage of Sendai virus in dcAMP-treated PCKC resulted in gradual decrease of infectious yield and eventual loss of virus infectivity (FIGURE 3).

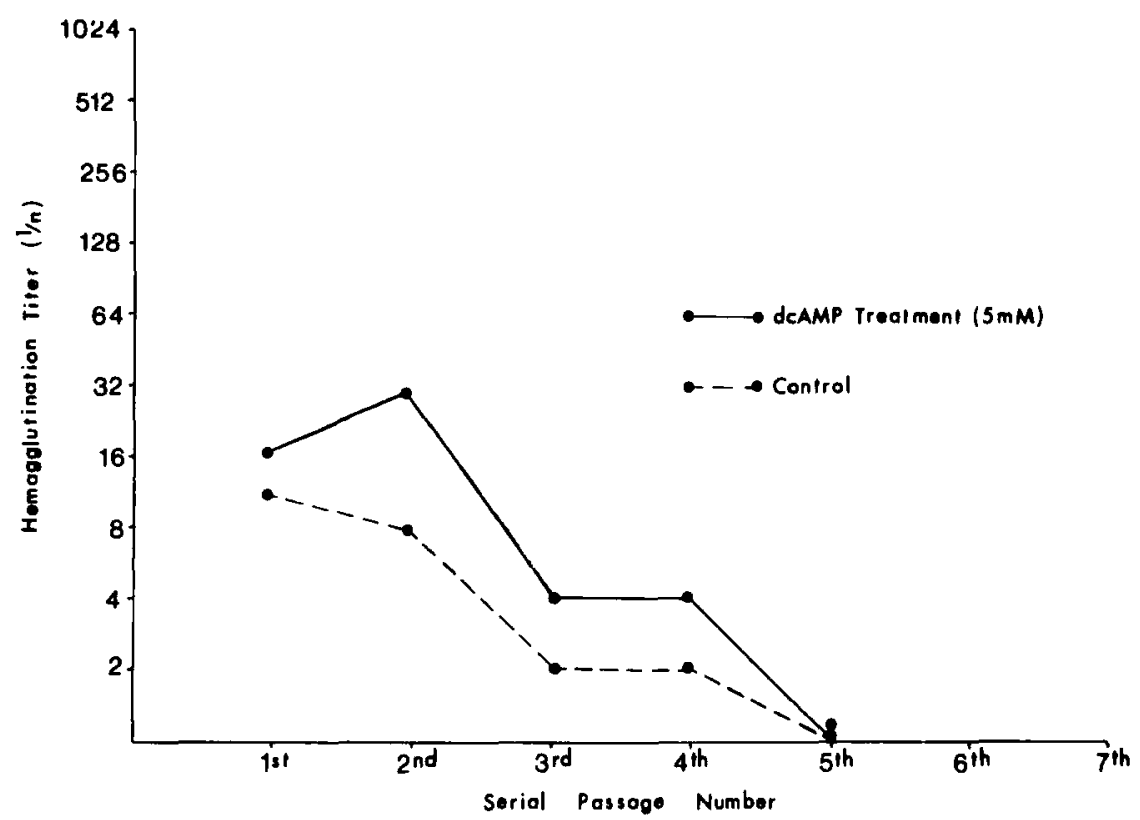

Figure 3. Effect of dcAMP on serial passage of Sendai (E9) virus in chick cells.

\section{Specificity of the Stimulation of Growth of Influenza Virus}

In order to test the specificity of the enhancing effect of dcAMP on influenza viral replication in $\mathrm{PCKC}$, a number of related compounds known to raise cellular cAMP levels by actions on the catalytic formation or breakdown of the cyclic nucleotide were used in serial passage of influenza virus-infected cells in order to duplicate the results obtained with dcAMP. The results presented in FIGUREs 4 and 5 indicate that the effects on enhanced viral synthesis is specific to accumulation of cellular cAMP. 


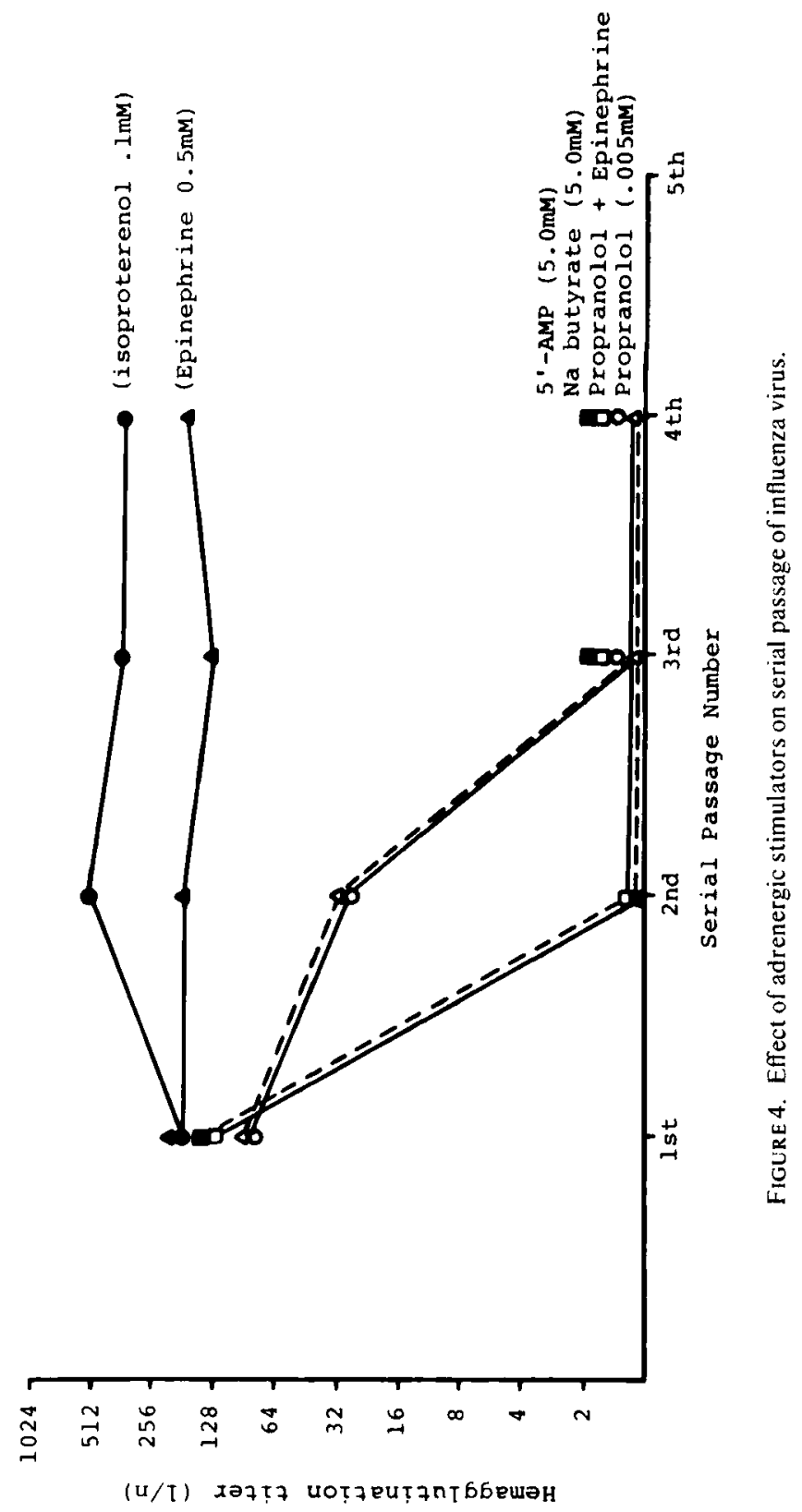




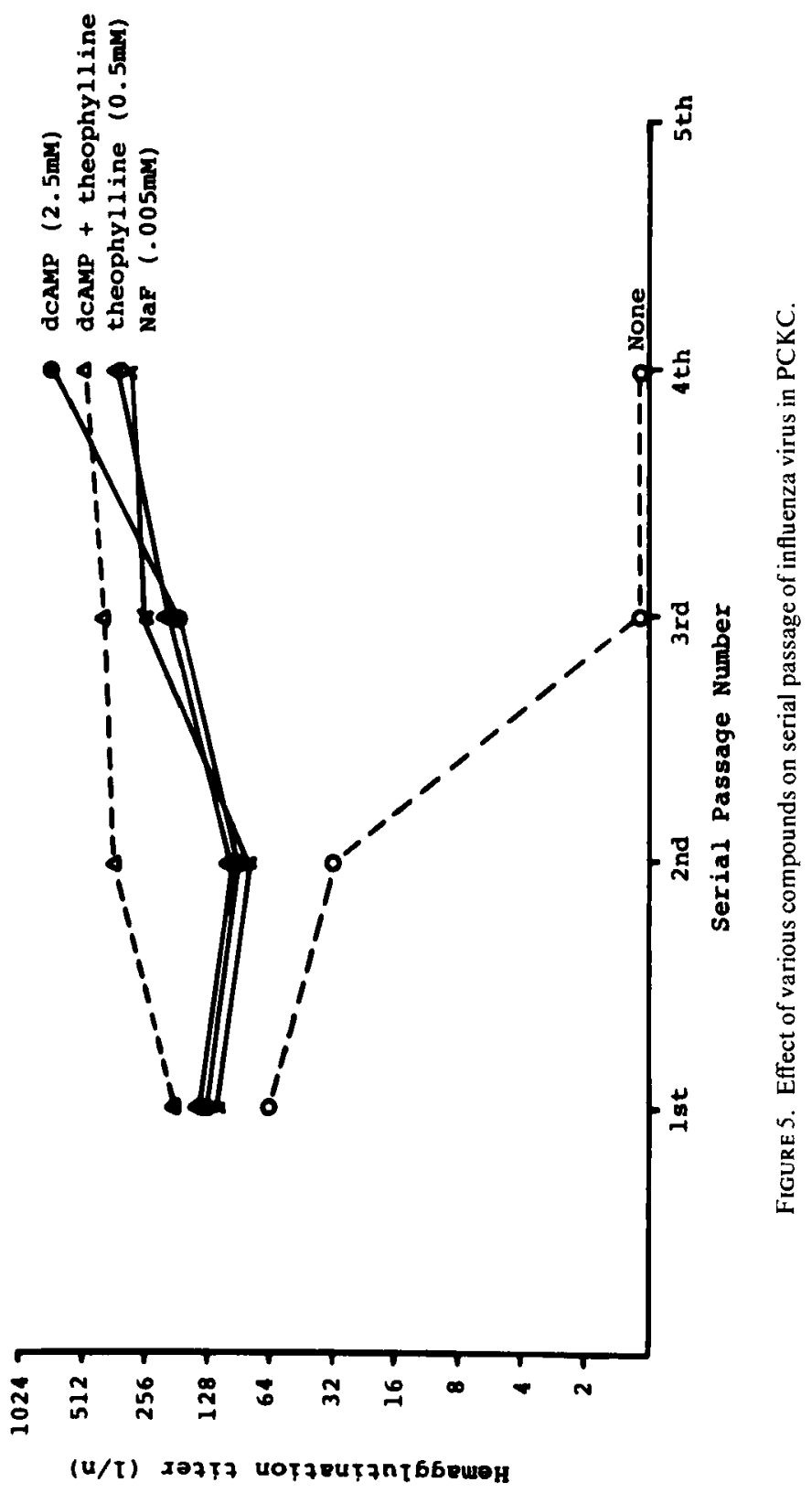


Compounds known to raise cellular cAMP levels by actions on the catalytic formation or breakdown of the cyclic nucleotide were utilized in serial passage experiments in attempts to duplicate the results obtained with dcAMP. Rapid serial passages of the A/AA/6/60- $\mathrm{H}_{2} \mathrm{~N}_{2}$ strain of influenza virus were initiated as described previously. Solutions of test agents made in double strength Eagle's medium were added at the time of infection, and cells were incubated in their presence for $48 \mathrm{hr}$. Results obtained indicate that the effect on enhanced viral growth is one mediated by cellular cAMP.

The adenyl cyclase of many cell systems generates an accumulation of endogenous cAMP as a result of stimulation by catecholamines and sodium fluoride. $^{13}$ Inhibition of phosphodiesterase catalyzed cellular cAMP breakdown by methylxanthine treatment accomplishes the same effect. ${ }^{14}$ Theophylline and sodium fluoride treatment of PCKC resulted in an effect on virus titer similar to that of addition of dcAMP to cell cultures. Furthermore, the presence of theophylline was shown, within the limits of this system, to potentiate the effect of $2.5 \mathrm{mM}$ dcAMP on the outcome of infection. An adenyl cyclase response to catecholamine stimulation has been documented by Sutherland and Robinson ${ }^{15}$ in a variety of cells. The adrenergic character of the response has been established. ${ }^{16}$ The net effect of the $\beta$-adrenergic agonist isoproterenol and the mixed stimulator epinephrine in the infected PCKC was an acceleration of infectious yield in serial passage. A similar effect of epinephrine was shown on $\mathrm{SV}_{40}$-transformed WI-38 fibroblasts, where it was far in excess of that of normal WI-38. ${ }^{17}$ The competitive interaction between epinephrine and the $\beta$-adrenergic antagonist propranolol has been studied in detail by Biel and Lum. ${ }^{18}$ Recently, propranolol has been utilized to abolish the $\beta$-adrenergic stimulation of human adipocyte lipolysis by epinephrine. ${ }^{2}$ The present study has shown that enhancement of influenza yield during serial passage in epinephrine-treated PCKC could also be abolished by treatment with propranolol. Propranolol itself did not promote the persistence of influenza under similar conditions. Of interest is the fact that although no $\alpha$-adrenergic antagonist was studied, other experimenters have shown a potentiation of the stimulatory action of epinephrine utilizing the $\alpha$-adrenergic blocking agent phentolamine. ${ }^{20}$

No similar effect on influenza biosynthesis in the PCKC could be detected using sodium- $N$-butyrate or 5 -AMP at several concentrations. In addition, another cyclic nucleotide guanosine $3^{\prime}: 5^{\prime}$-cyclic monophosphate (cGMP) did not alter the course of infection in PCKC since no enhancement or inhibition was observed with this compound.

\section{The Influence of dcAMP on MaCromolecular Synthesis of ViRUS-INFECTED CELLS}

The nature of the enhancement of influenza virus replication by treatment of PCKC with dcAMP was investigated and related to the overall rates of RNA and protein synthesis. ${ }^{20}$ Figure 6 shows the results of a typical experiment in which TCA-precipitable radioactivity of dcAMP-treated infected and uninfected cultures is presented as a percentage of corresponding infected or uninfected control cell levels. Total infected cell protein synthesis was observed to undergo a period of enhancement roughly between 4 and $8 \mathrm{hr}$ p.i. in the presence of dcAMP. In contrast, incubation of PCKC with dcAMP results in a short-lived burst of protein synthesis immediately after addition of compound to the untreated cells. Thereafter, protein synthesis is affected only slightly. The timing of enhancement of infected-cell protein 


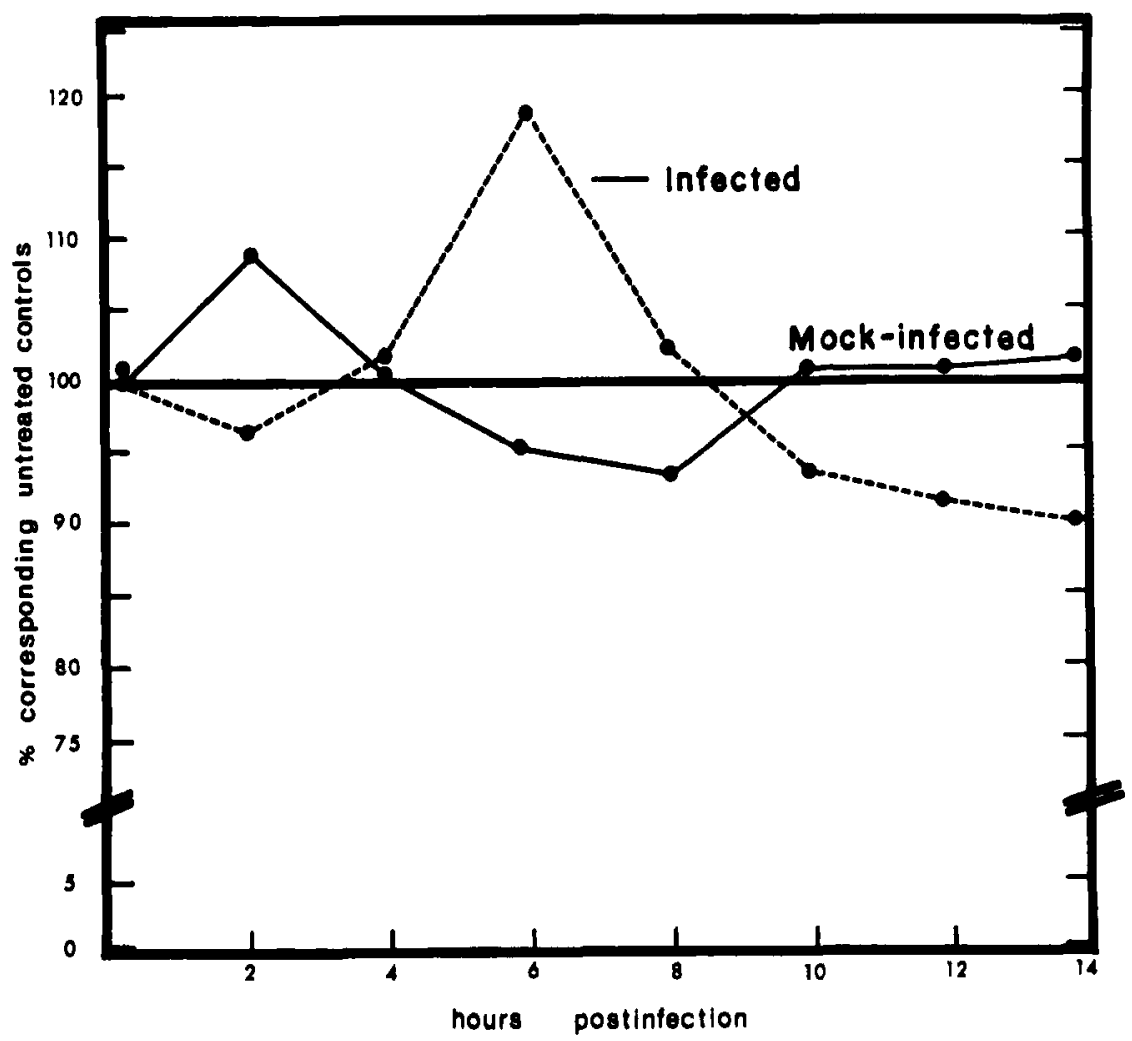

FIGURE 6. Incorporation of $\left[{ }^{3} \mathrm{H}\right]$-valine into acid-precipitable material of PCKC. Data expressed as percentage of corresponding untreated control values; $\bullet-\cdots \bullet$, infected; $\bullet-\ldots$. mock-infected.

synthesis resembles the pattern obtained from use of dcAMP in the biological studies of time course of treatment with the cyclic nucleotide and resultant virus yield.

Monitoring acid-soluble radioactivity indicated that dcAMP treatment resulted in an initial increase in precursor uptake immediately following addition of compound, and preceding the period of enhancement seen in TCA-precipitable radioactivity. A consistently slight enhancement of precursor uptake throughout infection is characteristic of infected and uninfected cell incubated with dcAMP.

From the pattern of protein synthesis observed, it is unlikely that enhancement of synthesis is due simply to alteration of cell permeability to precursors early in infection but to specific enhancement of virus-induced protein synthesis.

The effect of treatment upon RNA synthesis of infected cells was investigated in a similar manner. In both infected and noninfected cultures, a pattern of depression of both uridine incorporation into TCA-precipitable material and uridine uptake can be observed. Data in FIGURE 7 is given as percentages of corresponding untreated control cultures as before. The inhibition of uridine incorporation into RNA of infected cells was never seen to be of the extent observed in the uninfected cultures. Because close parallels were always obtained between uridine incorporation and 
uptake, it seemed apparent that the decreased RNA synthesis of both uninfected and infected PCKC was due to the interference with the uptake of uridine affected by the dibutyryl compound. This observation has substantiation elsewhere in the literature. ${ }^{20}$ It is not clear at this state, whether the phenomenon of viral stimulation observed can be related only to specific enhancements of viral proteins, but it was demonstrated recently that exogenous addition of compounds that enhance intracellular cAMP also enhances antibody synthesis without elevating DNA and RNA synthesis. ${ }^{21}$

\section{EFFECTS OF dcAMP ON GROWTH OF Herpesvirus hominis TYPE 2}

Experiments were designed to study the effect of dcAMP on the growth of a DNA virus, Herpesvirus hominis (HVH) Type 2 (Curtis strain) in PCKC. This virus was obtained from Dr. A. Nahmias (Emory University, Atlanta, Ga.) and a plaquepurified pool was made in chick kidney cells incubated at $35^{\circ} \mathrm{C}$. The design of this study was the same as presented earlier with influenza virus, in which dcAMP-

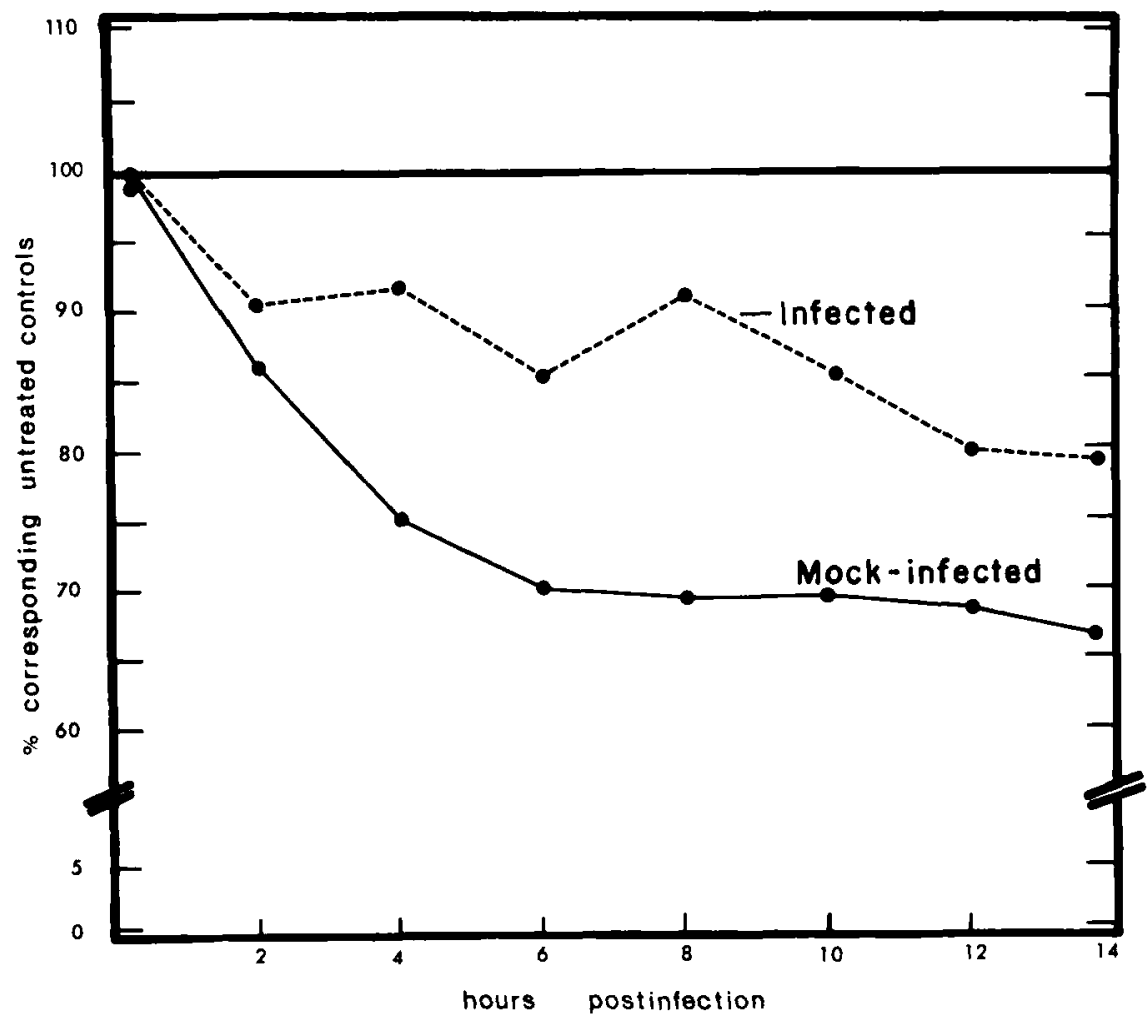

FIGURE 7. Incorporation of $\left[{ }^{3} \mathrm{H}\right]$-uridine into acid-precipitable material of PCKC. Data expressed as percentage of corresponding untreated control values; $\bullet-.-\bullet$, infected; $\bullet-\ldots$, mock-infected. 
treated and untreated cells were infected with $10^{-3}$ dilution of HVH Type 2 (titer $10^{\mathrm{B}, 0} \mathrm{PFU} / \mathrm{ml}$ ). Adsorption and maintainance media consisted of $2 \times$ Eagle's basal solution supplemented with $2 \%$ horse serum. The infectious yield was determined after $48 \mathrm{hr}$ p.i. by titration of the harvest in tissue culture (TCID $\mathrm{T}_{50}$ ), or in 12-gm Swiss mice (Webster strain) using intracerebral inoculation $\left(\mathrm{MLD}_{50}\right)$. The results presented in TABLE 5 show a definite inhibition in the growth of infectious virus in treated cells as measured in mice or in cell culture by cytopathic changes. Furthermore, Herpesvirus hominis was lost after serial passages in treated cells, while in untreated cells, growth of virus was sustained. The inhibition of herpesvirus replication and cytopathic changes in PCKC by dcAMP could be attributed to induction of latency by high levels of intracellular cAMP. This assumption is based on the fact that the treatment of HVH-infected cells with dcAMP initiates only a few foci of cellular degeneration as compared to untreated infected cells where complete cytolysis was evident. In addition, the treated cells after prolonged incubation seem to have recovered from herpesvirus infection and appear to have similar morphology and density as control cells. Hence, it is possible to speculate that this inhibition can be related to the action of cyclic nucleotides promoting latency of a DNA virus since it is well documented that dcAMP markedly inhibited the induction of DNA synthesis by Type 12 adenovirus. ${ }^{22}$ Replication of adenovirus Type 2 was also inhibited

TABLE 5

EfFect of dcAMP on Growth of Herpesvirus hominis Type 2 in Chick Cells

\begin{tabular}{ccccc}
\hline \hline & \multicolumn{3}{c}{$\begin{array}{c}\text { Mice } \\
\text { Infectivity Titer* in }\end{array}$} \\
& \multicolumn{2}{c}{$\begin{array}{c}\text { Tissue Culture } \\
\left(\text { LD }_{50} / 0.03\right)\end{array}$} & \multicolumn{2}{c}{$\begin{array}{c}\text { TCID } \\
\text { E0 } / m l)\end{array}$} \\
Experiment No. & Treated & Untreated & Treated & Unt reated \\
\hline I & $10^{1.0}$ & $10^{2.3}$ & $10^{1.7}$ & $10^{4.7}$ \\
II & $10^{1.3}$ & $10^{2.7}$ & $10^{1.7}$ & $10^{4.0}$ \\
\hline \hline
\end{tabular}

*The yield of 48 hours postinfection from dcAMP-treated PCKC and controls was titrated simultaneously in mice and tissue culture.

by dcAMP in three cell culture lines. ${ }^{23}$ The induction of cellular DNA synthesis and of adenovirus specific tumor antigen by oncogenic adenovirus can also be inhibited by addition of dcAMP. In addition, in isolating mutants of Salmonella typhimurium defective in adenyl cyclase or in cAMP-receptor protein, we noted that the mutants were lysogenized at reduced frequency by phage P22. ${ }^{24}$ Lysogenation could be stepped-up to normal values by the addition of $1 \mathrm{mM}$ cAMP to the culture of adenyl cyclase deficient mutants but not with others. An analogous mechanism was noted to function in the $E$. coli phage system in which cAMP and a catabolite-activator protein are evidently required directly to initiate transcription of cataboliterepressed host cistrons as well as of phage genes necessary for establishing latency. ${ }^{19}$

\section{SUMMARY}

The yield of influenza virus from infected primary chick kidney cells (PCKC) was enhanced by treatment with dcAMP. In addition, rapid serial passages of the virus in treated chick cells at low multiplicities did also exhibit maintenance of high virus titers. The action of dcAMP upon viral replication could be reproduced in the chick cells by treatment with various agents known to increase intracellular cAMP levels by differing mechanisms. Furthermore, an adrenergic component in the activation of 
the PCKC cAMP system is indicated by the effects of certain catecholamines on influenza virus yield. Dibutyryl cAMP was shown in the same host to inhibit multiplication of Herpesvirus hominis Type 2, and its effect on other cell-virus systems indicates that its action consists of some host-specific element(s).

\section{REFERENCES}

1. Sutherland, E. W. \& T. W. Rall. 1960. The relation of adenosine-3'-phosphate and phosphorylase to the actions of catecholamines and other hormones. Pharmacol. Rev. 12: 265 .

2. Burns, T. W., P. E. Langly, G. A. Robson. 1971. Adrenergic receptors and cAMP in the regulation of human adipose tissue lipolysis. Ann. N.Y. Acad. Sci. 185: 115.

3. Pastan, I. \& G. S. Johnson. 1974. Cyclic AMP and the transformation of fibroblasts. Adv. Cancer Res.: 303.

4. Garren, L. D., G. N. Gill \& G. Walton. 1971. Isolation of a receptor for cAMP from adrenal cortex: Role of receptor on mechanisms of action of cAMP. Ann. N.Y. Acad. Sci. 185: 210.

5. TEWS, J. K., N. A. Woodcock \& A. E. Harper. 1970. Stimulation of amino acid transport in rat liver slices by epinephrine, glucagon, and adenosine $3^{\prime}, 5^{\prime}$-monophosphate. J. Biol. Chem. 245: 3026.

6. HaIT, G., J. KYPSON \& R. MassiH. 1972. Amino acid incorporation into myocardium: Effect of insulin, glucagon, and dibutyryl 3',3'-AMP. Amer. J. Physiol. 222: 404.

7. Somlyo, A. P., A. V. Somlyo \& N. Friedmann. 1971. Cyclic adenosine monophosphate, cyclic guanosine monophosphate, and glucagon: Effects of membrane potential and ion fluxes in the liver. Ann. N.Y. Acad. Sci. 185: 108.

8. MaAssab, H. F. 1970. The propagation of multiple viruses in chick kidney cultures. Proc. Nat. Acad. Sci. 45: 1035.

9. Johnson, G. S. \& I. PASTAN. 1972. Role of cAMP in regulation of morphology and growth of transformed and normal fibroblasts. J. Nat. Cancer Inst. 48: 1377.

10. HeErsch, J. N., S. A. Fedak \& G. D. Aurbach. 1970. The mode of action of dibutyryl adenosine $3^{\prime}, 5^{\prime}$-monophosphate on bone tissue in vitro. J. Biol. Chem. 246: 6770.

11. Tihon, C. \& M. Green. 1973. Cyclic AMP-amplified replication of RNA tumour viruslike particles in chinese hamster ovary cells. Nature New Biol. 244: 227.

12. YANG, T. J. \& N. W. WANG. 1974. Dibutyryl cyclic AMP: Increase in viral particles associated with morphologic changes in leukemic cells. Exp. Molec. Pathol. 20: 147.

13. BURKE, G. 1970. Comparison of thy rotropin and sodium fluoride effects on thyroid adenyl cyclase. Endocrinology 86: 346.

14. Butcher, R. W. \& E. W. Sutherland. 1962. Adenosine 3':5'-phosphate in biological materials. I. Purification and properties of cyclic $3^{\prime}: 5^{\prime}$-nucleotide phosphodiesterase and use of this enzyme to characterize adenosine $3^{\prime}: 5^{\prime}$-phosphate in human tissues. J. Biol. Chem. 237: 1244.

15. Sutherland, E. W. \& G. A. Robinson. 1966. Metabolic effects of catecholamines: A. The role of cyclic $3^{\prime}, 5^{\prime}$-AMP in responses to catecholamines and other hormones. Pharmacol. Rev. 18: 145.

16. KNO, J. F. \& W. N. KNo. 1973. Regulation by beta-adrenergic receptor and muscarinic cholinergic receptor activation of intracellular cyclic AMP and cyclic GMP levels in rat lung slices. Biochem. Biophys. Res. Commun. 55: 660 .

17. Kelly, L. A., M. S. Hall \& R. W. Butcher. 1974. Cyclic adenosine 3':5'-monophosphate metabolism in normal and $\mathrm{SV}_{40}$-transformed WI-38 cells. J. Biol. Chem. 294: 5182 .

18. BIEL, J. H. \& B. K. B. LUM. 1966. The beta-adrenergic blocking agents; pharmacology and structure-activity relationships. Prog. Drug Res. 10: 46.

19. Grodzicker, T., R. R. ArditTI \& H. EIS EN. 1972. Establishment of repression by lambdoid phage in catabolite activator protein and adenylate cyclase mutants of Escherichia coli. Proc. Nat. Acad. Sci. U.S.A. 69 : 366. 
20. Hauschka, P. V., L. P. Everhart \& R. W. Rubin. 1972. Alteration of nucleoside transport of chinese hamster cells by dibutyryl adenosine $3^{\prime}: 5^{\prime}$-cyclic monophosphate. Proc. Nat. Acad. Sci. U.S.A.69: 3542.

21. Cook, R. G., A. B. Stavitsky \& M. D. Schoenberg. 1975. Regulation of the in vitro early anamnestic antibody response by exogenous cholera enterotoxin and cyclic AMP. J. Immunol. 114: 426.

22. ZimmermaN, J. E. \& K. RASKA. 1972. Inhibition of adenovirus type 12 induced DNA synthesis in G1-arrested BHK21 cells by dibutyryl adenosine cyclic 3':5'-monophosphate. Nature (New Biology) 239: 145.

23. BIRON, K. K. \& K. RASKA. 1973. Effects of dibutyryl-cAMP on adenovirus replication in different cell lines. Virology 56: 383.

24. Hong, J. S., G. R. Smith \& B. N. Ames. 1971. Adenosine 3':5'-cyclic monophosphate concentration in the bacterial host regulates the viral decision between lysogeny and lysis. Proc. Nat. Acad. Sci. U.S.A. 68: 2258. 